\title{
Glutathione depletion sensitizes cisplatin- and temozolomide-resistant glioma cells in vitro and in vivo
}

\author{
This article has been corrected since Online Publication and a corrigendum has also been published
}

\author{
CRR Rocha ${ }^{1}$, CCM Garcia ${ }^{1,3}$, DB Vieira ${ }^{1}$, A Quinet ${ }^{1}$, LC de Andrade-Lima ${ }^{1}$, V Munford ${ }^{1}$, JE Belizário² ${ }^{2}$ and CFM Menck ${ }^{\star, 1}$
}

Malignant glioma is a severe type of brain tumor with a poor prognosis and few options for therapy. The main chemotherapy protocol for this type of tumor is based on temozolomide (TMZ), albeit with limited success. Cisplatin is widely used to treat several types of tumor and, in association with TMZ, is also used to treat recurrent glioma. However, several mechanisms of cellular resistance to cisplatin restrict therapy efficiency. In that sense, enhanced DNA repair, high glutathione levels and functional p53 have a critical role on cisplatin resistance. In this work, we explored several mechanisms of cisplatin resistance in human glioma. We showed that cellular survival was independent of the p53 status of those cells. In addition, in a host-cell reactivation assay using cisplatin-treated plasmid, we did not detect any difference in DNA repair capacity. We demonstrated that cisplatin-treated U138MG cells suffered fewer DNA double-strand breaks and DNA platination. Interestingly, the resistant cells carried higher levels of intracellular glutathione. Thus, preincubation with the glutathione inhibitor buthionine sulfoximine (BSO) induced massive cell death, whereas $\mathrm{N}$-acetyl cysteine, a precursor of glutathione synthesis, improved the resistance to cisplatin treatment. In addition, BSO sensitized glioma cells to TMZ alone or in combination with cisplatin. Furthermore, using an in vivo model the combination of BSO, cisplatin and TMZ activated the caspase 3-7 apoptotic pathway. Remarkably, the combined treatment did not lead to severe side effects, while causing a huge impact on tumor progression. In fact, we noted a remarkable threefold increase in survival rate compared with other treatment regimens. Thus, the intracellular glutathione concentration is a potential molecular marker for cisplatin resistance in glioma, and the use of glutathione inhibitors, such as BSO, in association with cisplatin and TMZ seems a promising approach for the therapy of such devastating tumors.

Cell Death and Disease (2014) 5, e1505; doi:10.1038/cddis.2014.465; published online 30 October 2014

Malignant gliomas are the most common and aggressive type of primary brain tumor in adults. Current therapy includes surgery for tumor resection, followed by radiotherapy and/or concomitant adjuvant chemotherapy with temozolomide (TMZ) or chloroethylating nitrosoureas (CNUs). However, these protocols have limited success, and patients diagnosed with glioma have a dismal prognosis, with a median survival of 15 months and a 5-year survival rate of $\sim 2 \% .{ }^{1}$ Several molecular mechanisms for cell resistance to these agents have been described. Because both are alkylating agents, the repair enzyme $\mathrm{O}_{6}$-methylguanine-DNA methyltransferase (MGMT) is certainly a first barrier that is associated with increased tumor resistance. ${ }^{2,3}$ The p53 status has also been proposed to act in an opposite manner in glioma cell resistance to TMZ or CNUs. Although p53 mutation is shown to be more resistant to TMZ treatment, owing to the induction of cell death, ${ }^{4}$ the $\mathrm{p} 53$ protein protects glioma cells after CNU treatment, most likely by improving other DNA repair systems. ${ }^{5}$
Cisplatin is one of the most effective anticancer drugs and is used as a first-line treatment for a wide spectrum of solid tumors, such as ovarian, lung and testicular cancer, ${ }^{6}$ and it is used for adjuvant therapy in gliomas. ${ }^{7}$ Cisplatin is a molecule formed by one platinum ion that is surrounded by four ligands at the cis position: two chloride atoms and two amine molecules. The mechanism of action of cisplatin is mainly based on DNA damage. Once inside the cell, cisplatin becomes activated by the substitution of one or two chloride atoms by water, a process known as aquation. Owing to this process, the drug becomes positively charged and interacts with the DNA molecule, inducing the formation of DNA adducts. Activated cisplatin preferentially binds to purine bases in the nucleophilic N7 sites, where the majority of adducts occur between two guanines on the same strand, whereas $\sim 3-5 \%$ of cisplatin adducts react with purines at the opposite strands, forming interstrand crosslinks (ICLs). The DNA lesions, in turn, trigger a series of signal-transduction pathways, leading to cell-cycle arrest, DNA repair and apoptosis. ${ }^{8}$

\footnotetext{
${ }^{1}$ Department of Microbiology, Institute of Biomedical Sciences, University of São Paulao, Av. Prof. Lineu Prestes, 1374, São Paulo 05505-900, Brazil and ${ }^{2}$ Department of Pharmacology, Institute of Biomedical Sciences, University of São Paulo, Av. Prof. Lineu Prestes, 1374, São Paulo 05508-900, Brazil

*Corresponding author: CFM Menck, Department of Microbiology, Institute of Biomedical Sciences, University of São Paulo, Av. Prof. Lineu Prestes, 1374, São Paulo 05508-900, Brazil. Tel: +55 11 30917499; Fax: +55 11 30917354; E-mail: calsmah@yahoo.com.br

${ }^{3}$ Current address: Departament of Biological Sciences, Instituto de Ciências Exatas e Biológicas, Federal University of Ouro Preto, Ouro Preto, MG, Brazil

Abbreviations: ACNU, nimustine; BSO, buthionine sulfoximine; CNU, chloroethylating nitrosourea; CTR1, copper transport channel; DSBs, double-strand breaks; GSH, glutathione; GST, glutathione $S$-transferase; HCR, host-cell reactivation; ICL, interstrand crosslink; MGMT, $\mathrm{O}_{6}$-methylguanine-DNA methyltransferase; NAC, N-acetyl cysteine; NER, nucleotide excision repair; $\left(0^{6} \mathrm{MeG}\right), 0^{6}$-methylguanine; TMZ, temozolomide

Received 09.6.14; revised 17.9.14; accepted 22.9.14; Edited by A Oberst
} 
Although relatively efficient, resistance to cisplatin, either intrinsic or acquired, during cycles of therapy is common, and overcoming tumor resistance remains the major challenge for cisplatin anticancer therapy. Cellular cisplatin resistance is a multifactorial phenomenon that may include decreased drug uptake, enhanced DNA repair capacity and higher glutathione (GSH) concentration. ${ }^{9}$

GSH is a highly abundant, low-molecular-weight peptide in the cell, and it is well known for its critical importance in maintaining the cellular oxidative balance as a free radical scavenger. Additionally, GSH has a protective role against xenobiotic agents once its highly reactive thiol group binds and inactivates those agents. In fact, the GSH content and glutathione $S$-transferase (GST) have long been associated with cisplatin resistance in numerous cell lines and tumor tissues. $^{9-11}$

Considering these possible pathways, it is not clear, however, which one determinates cisplatin resistance in glioma cells. Aiming to better understand the molecular mechanisms of resistance to this drug, four human glioma cell lines with different p53 status were investigated. We showed that cellular resistance was found to be independent of p53 as well as of the DNA repair capacity of the cells. On the other hand, the GSH levels within the cell were shown to act as a decisive resistance barrier to cisplatin, reducing the induction of DNA damage in the treated cells. Also, both in
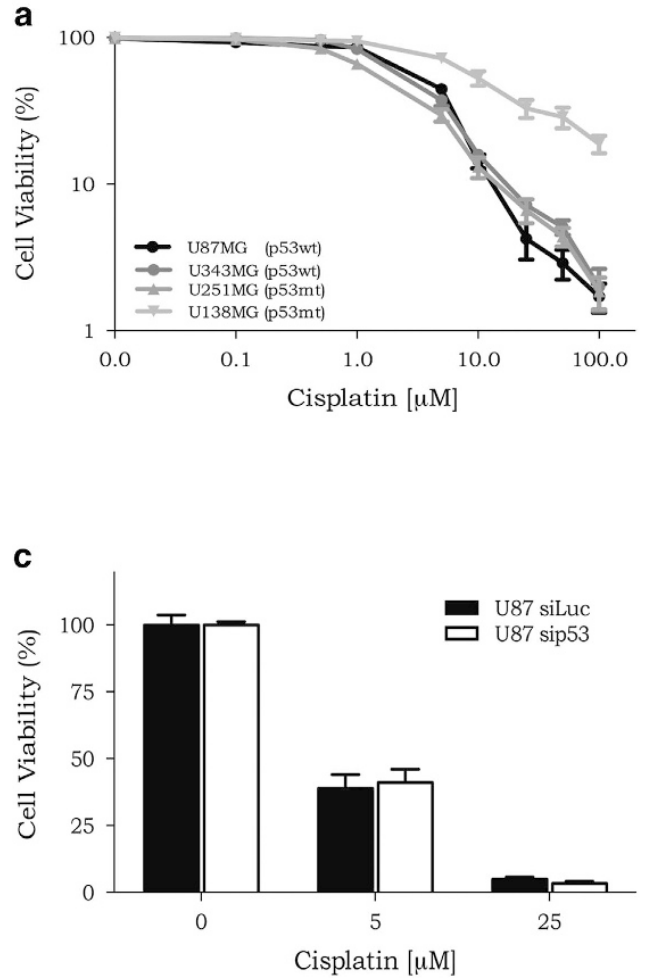

an in vitro and in vivo model depletion of GSH by an inhibitor (buthionine sulfoximine, BSO) sensitized the glioma cell lines to cisplatin. Interestingly, BSO also potentiated TMZ cytotoxicity. Thus, combination with BSO, cisplatin and TMZ turned out to be an extremely powerful approach to improve cytotoxicity in glioma, thus providing an exciting alternative for glioma treatment.

\section{Results}

The p53 status alone is not sufficient to determine cisplatin sensitivity in glioma cells. It has been demonstrated that functional p53 has an opposing effect in glioma treated with $\mathrm{TMZ}$ or nimustine (ACNU). ${ }^{4,5}$ To extend these observations to cisplatin treatment, the cell sensitivity was evaluated in glioma cell lines with different p53 status: U87MG and U343MG (p53wt) and U251MG and U138MG (p53mt). All four lineages were incubated with increasing doses of cisplatin, and, after $120 \mathrm{~h}$ of treatment, the cellular viability and apoptotic levels were determined. It was observed that U138MG (p53mt) cells were less sensitive to cisplatin treatment, whereas the other lineages displayed quite similar survival rates, despite their different p53 status (Figure 1a). In agreement, the analysis of the sub-G1 populations, a common methodology used for DNA fragmentation analysis because of apoptosis, after cisplatin exposure b

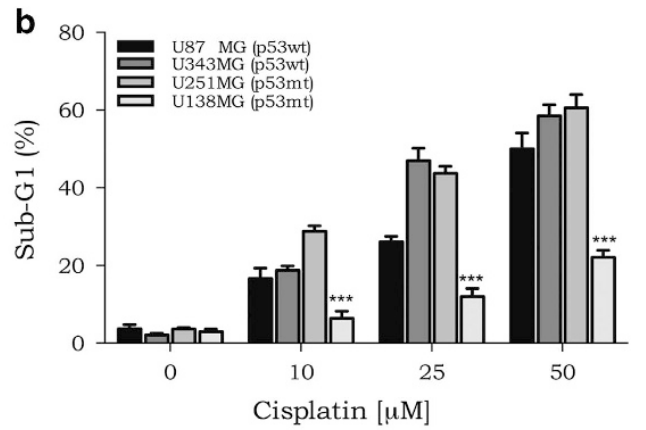

d

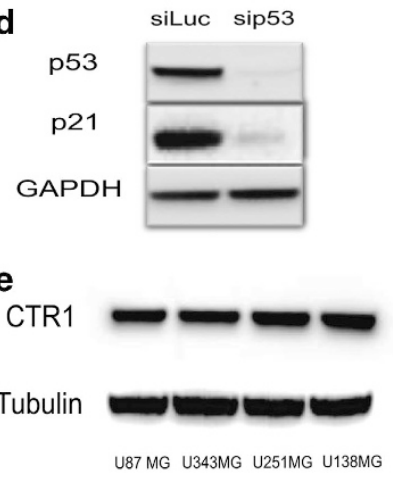

Figure 1 Cellular viability of glioma cells after exposure to cisplatin. (a) A dose-response curve of four glioma cell lines treated with increasing concentrations of cisplatin $(0.01-100 \mu \mathrm{M})$ and analyzed after $120 \mathrm{~h}$ treatment using the XTT assay. (b) A dose-response curve of the apoptotic fraction of glioma cells treated with increasing doses of cisplatin after $120 \mathrm{~h}$ of incubation time, analyzed as the sub-G1 population levels using flow cytometry of PI-stained nuclei. Values are mean \pm S.E.M. of three independent experiments, ${ }^{\star} P<0.05,{ }^{* \star} P<0.01$ and ${ }^{* * \star} P<0.001$. (c and d) Transient transfection of siRNA targeting the $p 53$ gene was able to knockdown the expression of this protein, and also reduced p21 expression, although it caused no effects on the sensitivity of these cells to cisplatin treatment. (e) Expression of CTR1 protein was analyzed by specific antibodies in whole-cell preparations using western blot analysis. Tubulin was used as loading control. The results in $\mathrm{E}$ and $\mathrm{F}$ are representative of one out of three experiments with comparable results 
indicated lower levels for the U138MG cell line (Figure 1b). On the other hand, U251MG (p53mt) displayed a high sensitivity upon cisplatin treatment, indicated either by low cellular viability or high amounts of apoptotic cells (Figures 1a and b). In addition, when siRNA was used to silence p53 in U87MG cells, no effects were observed for cell survival after cisplatin treatment (Figures $1 \mathrm{c}$ and $\mathrm{d}$ ). Those results indicate that cell sensitivity to cisplatin is independent of the p53 status in glioma cells, and other resistance factors have a more decisive role in protecting cells from cell death induced by cisplatin.

Another import resistance mechanism displayed by some tumor cells is a reduced expression level of copper transport channel (CTR1), which is involved in cisplatin uptake. Thus, lower expression of this transport channel could decrease the amount of intracellular cisplatin and could explain the resistance of the U138MG cells. However, a western blot
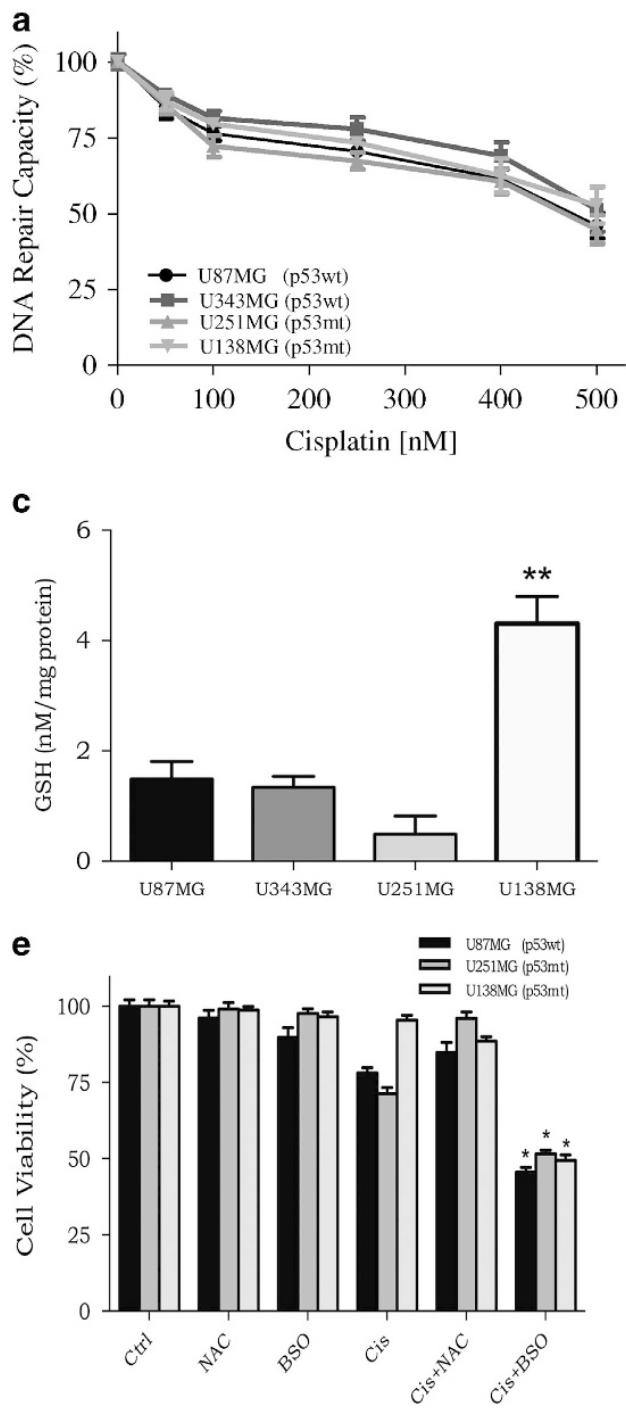

revealed that there is no difference in the expression of CTR1 protein in the four cell lines investigated (Figure 1e).

DNA repair is not involved in the cell resistance. To investigate whether U138MG resistance to cisplatin was because of an enhanced DNA repair capacity, host-cell reactivation ( $\mathrm{HCR})$ assays were performed using a firefly luciferase gene reporter. In this assay, cisplatin-treated plasmids are transfected into the cells, and the bioluminescence that is detected, owing to reactivation of luciferase expression, is directly related to the ability of cells to remove DNA damage. The results of the HCR assay showed no difference in the bioluminescence emission from the four cell lineages, which indicates that all cell lines have a similar DNA repair capacity to remove cisplatin lesions from the damaged plasmids (Figure 2a).
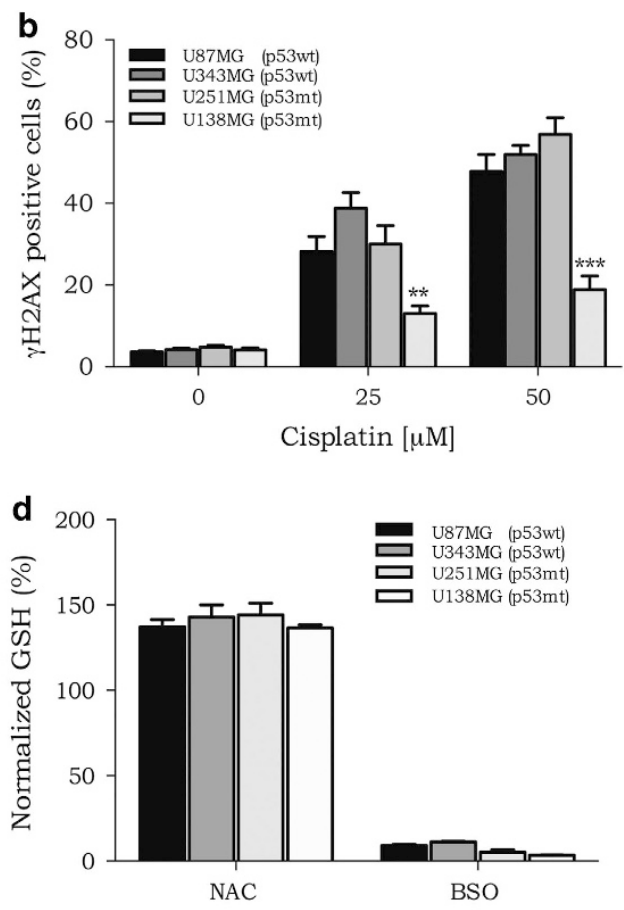

f

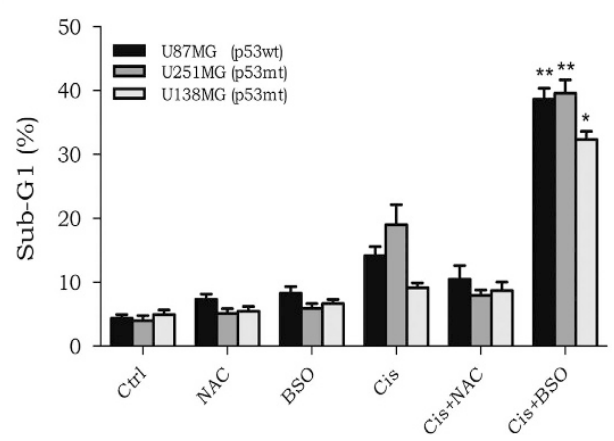

Figure 2 DNA repair capacity and GSH concentration in glioma cell lines. (a) HCR assay with a luciferase plasmid treated with increasing doses of cisplatin. (b) Quantification of $\gamma \mathrm{H} 2 \mathrm{AX}$-positive cells upon cisplatin treatment, as detected by flow cytometry. (c) Quantification of the basal intracellular GSH concentration in the four glioma cell lines. (d) Quantification of the intracellular GSH concentration after BSO or NAC incubation. (e) Cellular viability, as determined by the XTT assay, in cells treated with BSO or NAC combined with cisplatin. (f) The percentage of apoptotic cells, as measured by the sub-G1 population, after treatment with cisplatin in combination with BSO or NAC. Values are mean \pm S.E.M. of three independent experiments, ${ }^{*} P<0.05,{ }^{* *} P<0.01$ and ${ }^{* * *} P<0.001$ 
During the DNA replication process, ICL lesions can lead to the formation of double-strand breaks (DSBs), these trigger the phosphorylation of histone $\mathrm{H} 2 \mathrm{AX}(\gamma \mathrm{H} 2 \mathrm{AX})$, which has been widely used as a DSB marker. ${ }^{12,13}$ Our results pointed to a reduced percentage of $\gamma \mathrm{H} 2 \mathrm{AX}$-positive cells in the resistant cells (Figure $2 b$ ), indicating that fewer DSBs were generated in U138MG cells after being treated with cisplatin. Altogether, these data suggest that U138MG resistance cannot be attributed to an improved DNA repair capacity, but these cells were somehow submitted to lower amounts of DNA damage after cisplatin treatment, when compared with the other glioma cell lines.

Depletion of GSH circumvents the resistance to cisplatin. Detoxification of cisplatin could also explain the different sensitivity of these cell lines, as this would reduce the impact of this drug. GSH may promote cisplatin detoxification, once it can bind covalently to intracellular cisplatin, preventing it from reacting with DNA, its therapeutic target. In fact, quantification of the intracellular GSH concentration of glioma cells showed that U138MG exhibited the highest levels among the investigated cell lines (Figure 2c). To modulate the cellular GSH concentration, BSO and N-acetyl-cysteine (NAC) were tested. BSO is a known inhibitor of $\gamma$-glutamylcysteine synthetase, an essential enzyme for the synthesis of GSH, and NAC is a GSH precursor, which increases the production of $\mathrm{GSH}$ within cells. As observed in Figure $2 \mathrm{~d}$, incubation with NAC or BSO for $18 \mathrm{~h}$ promoted a substantial increase or depletion, respectively, in the GSH levels of all four cell lines.

Aiming to investigate the role of GSH in cisplatin resistance, glioma cells were incubated with BSO $(100 \mu \mathrm{M})$ or NAC (25 mM) for $18 \mathrm{~h}$ and then treated with cisplatin. BSO or NAC alone did not result in a decrease in cell viability or in a significant increase in apoptosis (Figures 2e and f). However, it was observed that $\mathrm{BSO}$ in combination with cisplatin sensitized all cell lines and, more importantly, BSO pretreatment was able to overcome U138MG cisplatin resistance (Figures $2 e$ and f). On the other hand, addition of NAC to the cell culture before cisplatin incubation was able to protect the cells from cell death triggered by cisplatin (Figure 2e). In addition, the percentage of apoptotic (sub-G1) cells was highly increased when cisplatin was combined with BSO, whereas NAC plus cisplatin protected the cells, with sub-G1 levels equivalent to those observed for the non-treated group (Figure 2f).

BSO increases cisplatin cytotoxicity by enhancing the production of DNA damage. To explore the mechanisms involved in inducing cell death, the levels of DNA damage were evaluated after combined treatment with BSO and cisplatin. In GSH-depleted samples, there is an increase of cisplatin-DNA adducts as detected by immunofluorescence or slot-blot (Figures $3 a$ and b). Furthermore, immunofluorescence staining of $\gamma \mathrm{H} 2 \mathrm{AX}$ was higher in the samples treated with BSO plus cisplatin (Figure $3 \mathrm{c}$ ). Flow cytometry analysis showed that BSO induced more than two times the amount of $\gamma \mathrm{H} 2 \mathrm{AX}$ staining, which demonstrated higher levels of DNA damage after cisplatin exposure in the presence of BSO (Figure 3d). Additionally, using quantitative PCR methodology, ${ }^{14}$ it was observed that the cisplatin plus BSO samples generated less PCR product, which indicates a higher amount of DNA lesions (Figure $3 \mathrm{e}$ ). In fact, estimation of the frequency of DNA lesions by Poisson distribution indicated that the combination of cisplatin and BSO induced more DNA damage than either drug alone (Figure 3f). Thus, it seems that depletion of GSH by preincubation with BSO enhances the amount of cisplatin that actually reaches the nuclear DNA, which, in turn, potentiates cisplatin-induced cytotoxicity.

Combination of BSO, cisplatin and TMZ: a high synergistic effect in vitro. The methylating agent TMZ is the firstline therapy drug used to treat glioma patients. As shown by previous work, ${ }^{15}$ BSO potentiates TMZ cytotoxicity (Figure 4a). In addition, as seen for BSO and cisplatin, the combination of BSO and $\mathrm{TMZ}$ greatly enhanced $\gamma \mathrm{H} 2 \mathrm{AX}$ positive cells (Figure 4b); in other words, GSH depletion potentiates TMZ killing effect because of the increase of DNA damage.

Based on the fact that the U138MG cell line was also resistant to $\mathrm{TMZ}$ treatment, ${ }^{4}$ the toxicity of glioma cells to BSO and cisplatin in combination with $\mathrm{TMZ}$ was investigated. To better verify a possible synergistic effect after drug combination, the U138MG cell line was treated with low doses of TMZ $(10 \mu \mathrm{M})$ and cisplatin $(1 \mu \mathrm{M})$ to cause minimal cellular toxicity. After $120 \mathrm{~h}$ of treatment with the drugs alone or in combination, cellular toxicity was determined. As it is clearly observed, the regimen treatment of $\mathrm{BSO}$, cisplatin and $\mathrm{TMZ}$ substantially reduced cellular viability and induced high levels of apoptosis, whereas pretreatment with NAC protected cells from toxicity mediated by cisplatin plus TMZ (Figures $4 \mathrm{c}$ and d). Thus, a synergistic effect $(P<0.001)$ was produced by combining $\mathrm{BSO}$, cisplatin and TMZ in glioma cell lines.

Combination of BSO, cisplatin and TMZ: a high synergistic effect in vivo. Finally, the synergistic effect of the combination of the three drugs that was observed in vitro was tested in vivo. Tumor progression was followed using in vivo bioluminescence imaging as this technology allows for the detection of tumor burden in a sensitive and noninvasive manner. For these experiments, glioma cell lines expressing luciferase were generated using lentivirus-derived vectors. In agreement with XTT and sub-G1 population assay data, the combination with BSO, cisplatin and TMZ was able to promote a strong increase in in vitro cell death, as detected by the low bioluminescence emission from the labeled cells (Figures $4 \mathrm{e}$ and f).

No tumor formation was detected upon inoculation of U138MG cells, and the same outcome was reported previously by others group. ${ }^{16}$ Therefore, U87-Luc was inoculated into the flanks of nude mice, and, after the tumor volume reached $\sim 100 \mathrm{~mm}^{3}$, the animals were divided into eight groups and treated with phosphate-buffered saline (PBS), BSO, cisplatin, TMZ or combinations of these drugs. The drug treatment regimen was one dose per day for three consecutive days and then once a week for 2 weeks. During the course of treatment, no severe side effects were observed, no animals died and there was no significant weight loss because of any combinatory drug treatment (Figure 6a). 
a

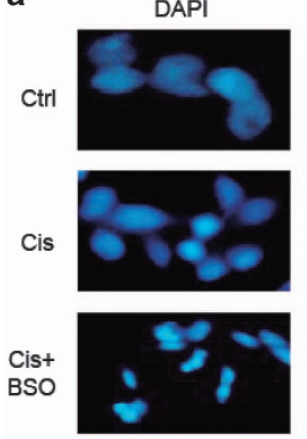

C

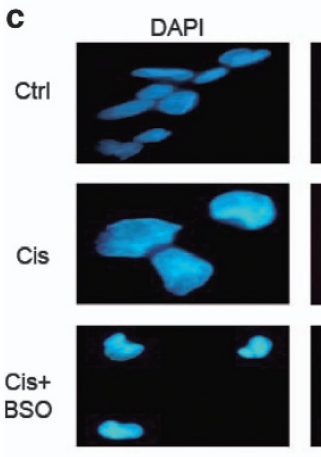

e
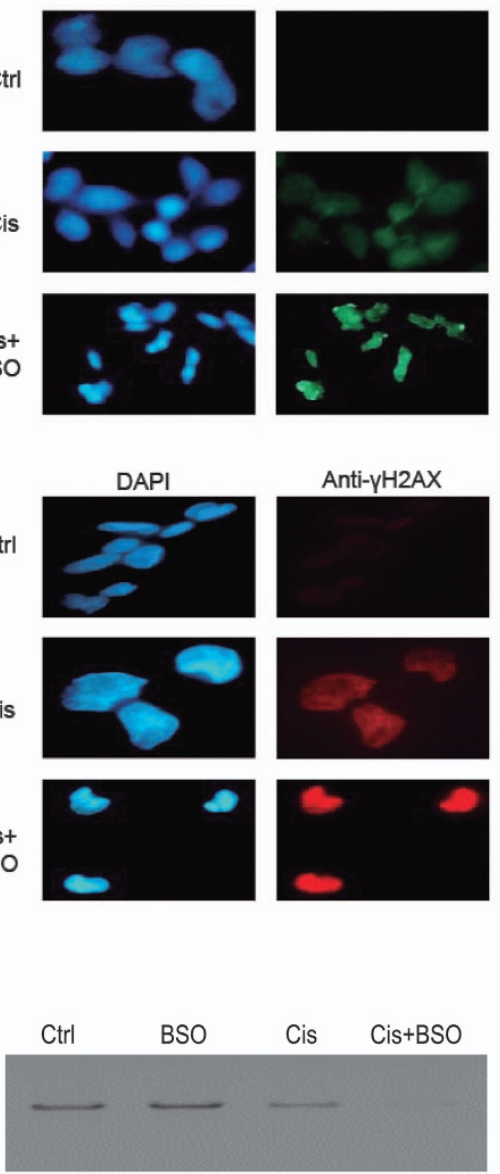

Anti-pH2AX
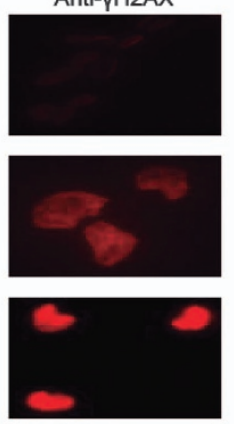

b
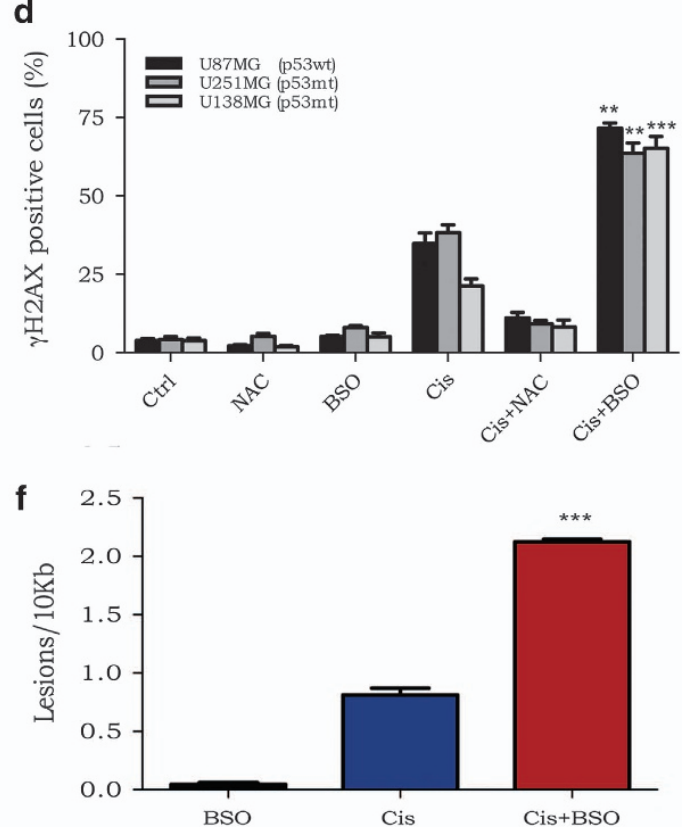

Figure 3 Detection of DNA damage in cisplatin-treated cells: cisplatin-DNA adducts and $\gamma \mathrm{H} 2 \mathrm{AX}$. (a) Immunofluorescence of cisplatin adducts in U138MG cells after treatment with cisplatin alone or in combination with BSO. (b) Immunodetection of cisplatin adducts by a slot-blot assay in U87MG and U138MG cells that were preincubated or not with BSO. (c) $\gamma \mathrm{H} 2 \mathrm{AX}$ immunofluorescence of samples treated with cisplatin treatment alone or with both drugs. Those results are representative of one out of three experiments with comparable results. (d) Quantification of the percentage of $\gamma \mathrm{H} 2 \mathrm{AX}$-positive cells by flow cytometry $24 \mathrm{~h}$ after treatment. (e) Representative quantitative PCR of damage measurements of samples treated with cisplatin only or in combination with BSO. (f) Quantification of DNA damage in treated cells. Values are mean \pm S.E.M. of three independent experiments, ${ }^{*} P<0.05,{ }^{* *} P<0.01$ and ${ }^{* * *} P<0.001$

Initially, to examine directly the ability of BSO, cisplatin and $\mathrm{TMZ}$ combinations to induce apoptosis in vivo, Z-DEVDaminoluciferin was used. This reagent is a prosubstrate that releases luciferin when it is cleaved by activated caspase- $3 / 7$. Three days after treatment, the animals were inoculated with Z-DEVD-aminoluciferin, and, $8 \mathrm{~h}$ later, luciferin was inoculated to measure tumor volume. Upon Z-DEVD-aminoluciferin bioluminescence normalization to the luciferin signal, the combination of BSO followed by cisplatin and TMZ significantly induced apoptosis in the nude mice bearing glioma tumors (Figures $5 a$ and b).

Remarkably, 2 weeks after the initial treatment, a strong inhibition on tumor growth progression was observed in the animal groups inoculated with BSO in combination with either cisplatin or with TMZ (Figure 6b). Moreover, this effect was much more pronounced when the animals were simultaneously inoculated with BSO, cisplatin and TMZ. In fact, at the end of treatment, the tumor volume of the control group (animals treated with PBS or BSO alone) was increased by $\sim 35$-fold in relation to that on the first day of treatment, whereas, in the group treated with the three drugs together, the average tumor volume was about the same as that at the beginning of treatment (Figures $6 b, c$ and e). For ethical reasons, animals with large tumor burden were killed. The key result is that, when using the combination of the three drugs, there was a significant increase in animal survival by about 3-fold change and notably a full tumor regression for one of the animals (Figure 6d).

\section{Discussion}

Nearly four decades after its FDA approval, the clinical benefits of cisplatin as an anticancer agent are unquestionable. However, tumor resistance to cisplatin still remains a huge challenge. Similarly, glioma treatment efficacy is limited and, as so, this disease remains incurable. Clearly, it is urgent 

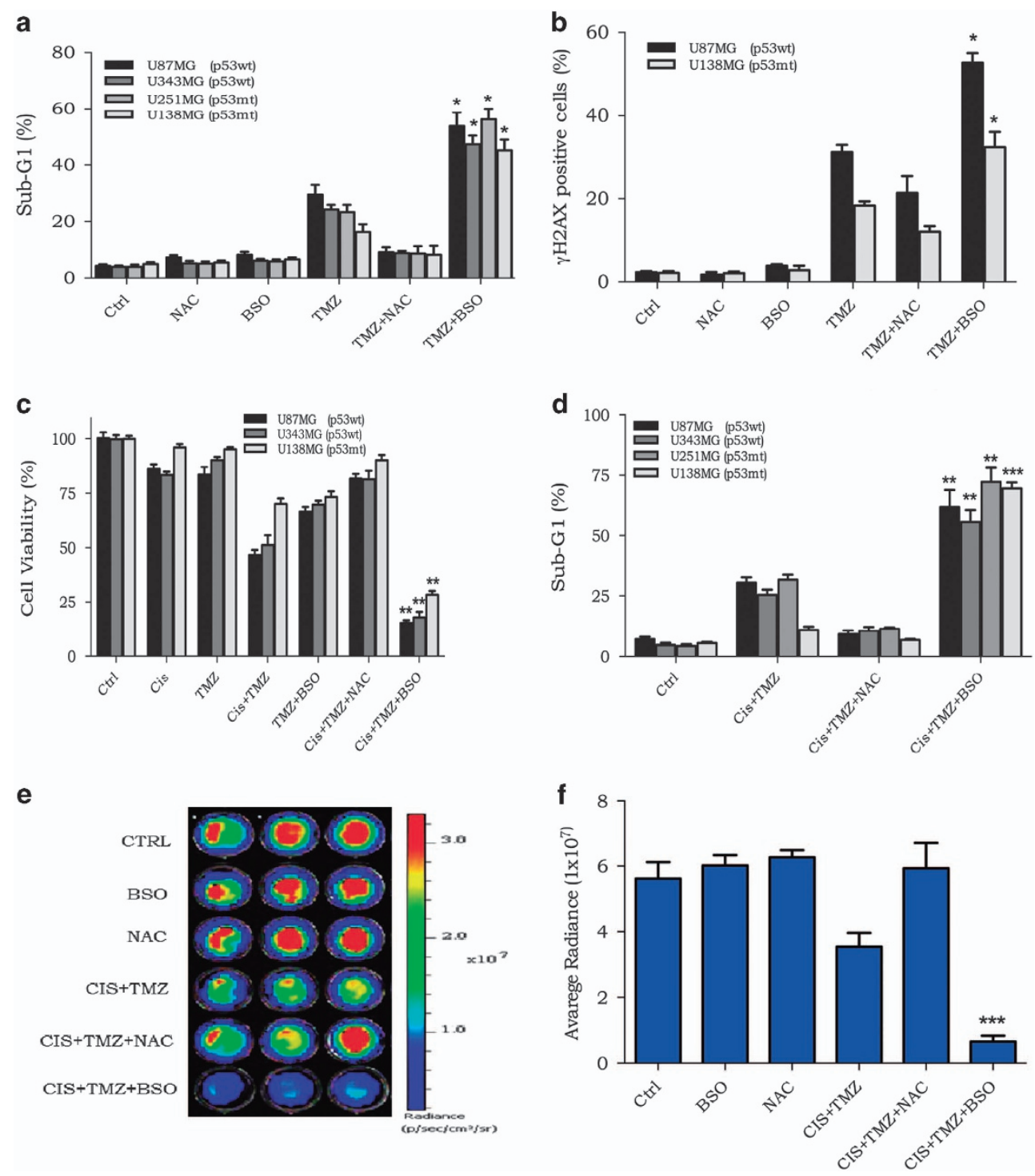

Figure 4 Cellular killing after exposure to BSO or NAC, cisplatin and TMZ. (a and b) Population of sub-G1 and $\gamma$ H2AX-positive cells upon treatment with BSO or NAC followed by TMZ. (c and d) U138MG cellular viability and apoptotic population after BSO or NAC followed by cisplatin and TMZ treatment. (e and f) Bioluminescence detection and quantification of luciferase-expressing glioma (U138-Luc) cells after the indicated treatment. Values are mean \pm S.E.M. of three independent experiments, ${ }^{*} P<0.05,{ }^{* *} P<0.01$ and ${ }^{* \star *} P<0.001$
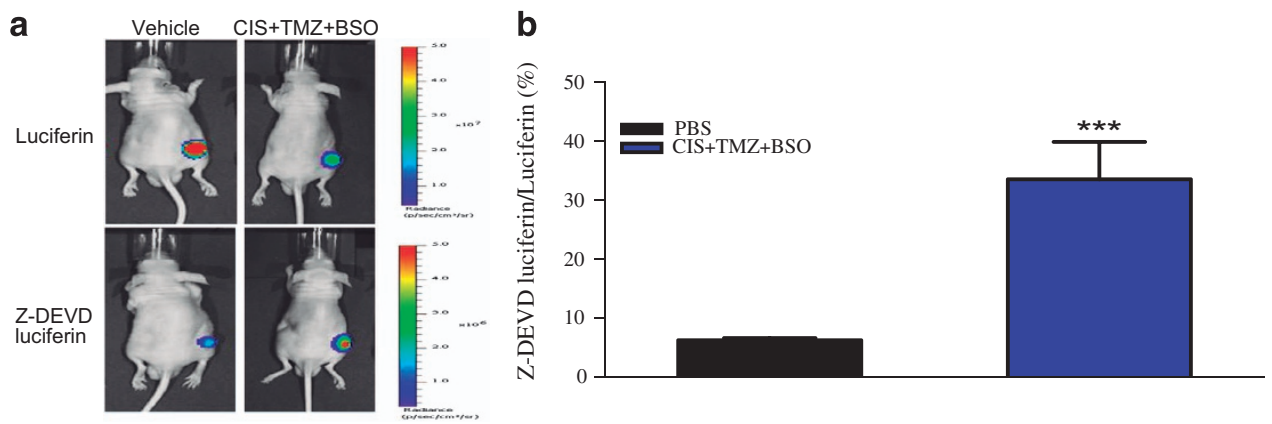

Figure 5 Induction of tumor cell apoptosis in vivo after treatment with cisplatin, TMZ and BSO. (a) Representative bioluminescence imaging of U87-Luc tumors after injection with Z-DEVD-aminoluciferin and luciferin. (b) Quantification of Z-DEVD-aminoluciferin bioluminescence signal, normalized by luciferin signal. Values are mean \pm S.E.M. of three independent experiments, ${ }^{*} P<0.05,{ }^{* \star} P<0.01$ and ${ }^{\star \star *} P<0.001$ 

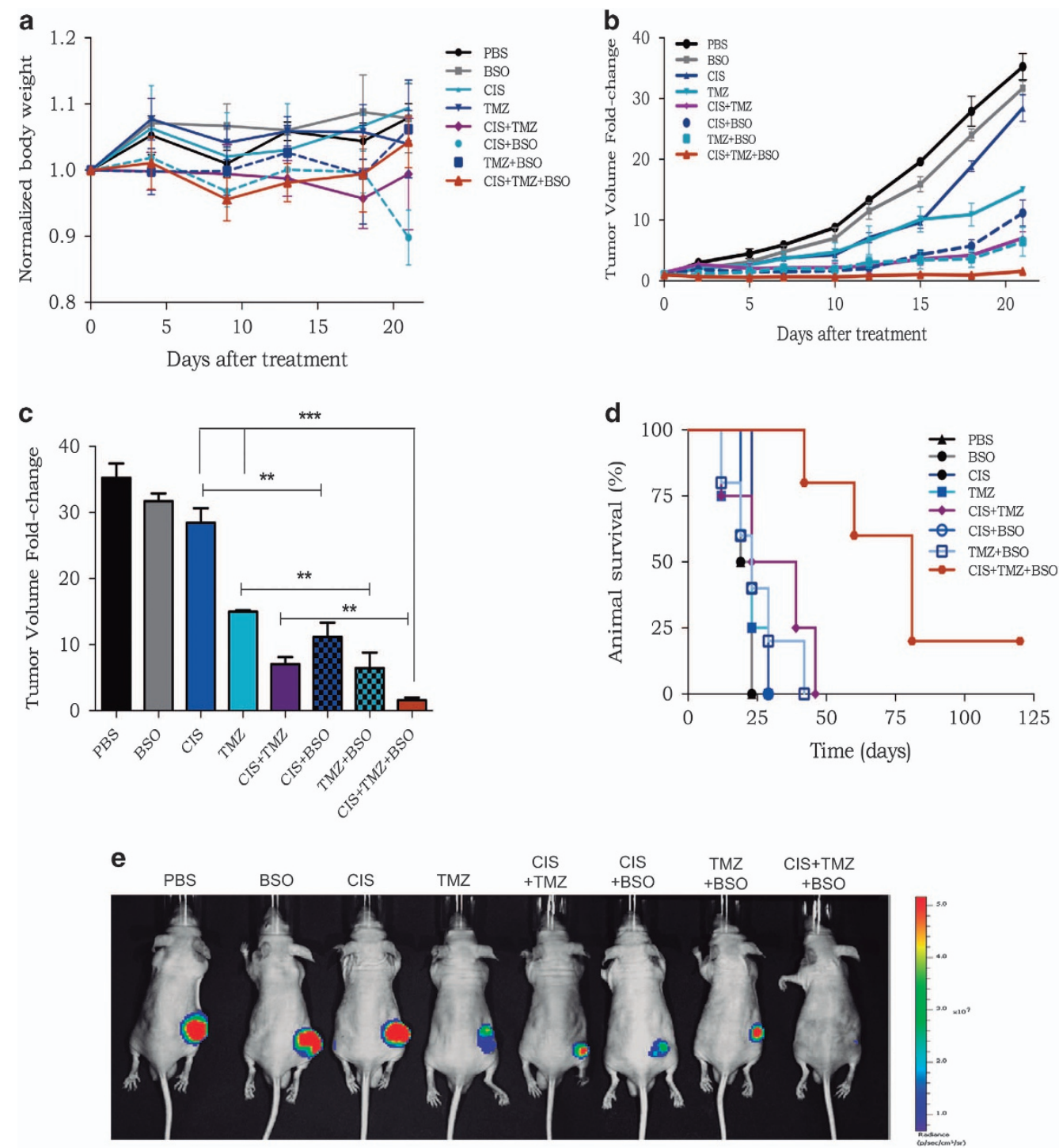

Figure 6 Tumor progression after treatment of animals with different chemotherapy combinations. (a) Time-course body weight progression was measured two times a week for 3 weeks in the eight treatment groups. (b) Time-course of U87-Luc tumor volume progression, as determined by caliper measurements. (c) Normalized tumor volume measured 21 days after the beginning of treatment. (d) Animal survival after treatment with different drug combinations, as indicated in the figure. Each point is the average of the tumor volume of five animals \pm S.E.M., ${ }^{*} P<0.05$, ${ }^{* *} P<0.01$ and ${ }^{* * *} P<0.001$. (e) Representative luciferin bioluminescence of U87-Luc tumor growth observed in animals treated with different drug combinations, as indicated, after 21 days

and necessary to search for an alternative and to improve the existing glioma therapy protocols.

In this study, several well-known mechanisms of resistance to cisplatin were investigated in glioma cell lines, with the aim to extend the knowledge of what factors are decisive in conferring resistance to this drug and search for alternatives to achieve a more favorable outcome for glioma patients.

The tumor suppressor gene p53 is commonly correlated with cellular sensitivity to several drugs used to treat cancer. In the majority of cases, it is reported that p53 mutation leads to cellular resistance to several drugs. However, there are conflicting data concerning cisplatin treatment and p53 status in glioma cell lines. ${ }^{17-19}$ In our study, using four distinct cell lines, two with wild-type p53 and two with mutated p53, no correlation between p53 status and cisplatin cellular sensitivity was observed: the cell viability, as determined by XTT assays, and apoptosis (sub-G1) results revealed that U251MG cells (p53mt) are highly sensitive to cisplatin, in contrast to U138MG, which is also a p53 mutant cell line. Additionally, p53 silencing did not cause any change in cell viability upon cisplatin treatment. Our results suggest that the absence of a functional p53 alone is not sufficient to confer cellular resistance to cisplatin in glioma cells.

Cisplatin adducts induce bulky alterations in the DNA structure that disturb DNA replication, leading to cell-cycle arrest and apoptosis. Those DNA adducts are recognized and repaired by the nucleotide excision repair (NER) pathway. Thus, NER activity is a key factor to promote cellular viability after cisplatin treatment. ${ }^{20}$ In that sense, an enhanced DNA repair capacity to address DNA lesions caused by cisplatin is another crucial cellular mechanism of resistance. Many studies have shown that resistant cells are able to remove 
cisplatin adducts at an increased rate ${ }^{21,22}$ or, conversely, demonstrated an association between high sensitivity and reduced DNA repair capacity. ${ }^{23}$ However, in the present work, no difference was observed in DNA repair capacity among the four cell lines, as indicated by the reactivation of damaged reporter gene expression. This implies that a more efficient DNA repair of cisplatin adducts cannot explain the U138MG drug resistance.

Cisplatin detoxification by GSH generally occurs by a reaction catalyzed by GST, in which GSH binds covalently to cisplatin and the formed GSH conjugates can be exported from the cells, preventing the drug from reaching its most important molecular target, DNA. ${ }^{24}$ Thus, DNA platination is strongly associated with cisplatin effectiveness; in other words, the higher amounts of DNA adducts, the better the treatment outcome. ${ }^{10,25}$ BSO has long been used as a potent inhibitor of GSH synthesis, and previous work has shown that, in fact, BSO was able to increase cytotoxicity in several cisplatin-resistant cell lines. ${ }^{9,26}$ It is also worth noting that BSO has been used in several clinical trials associated with anticancer drugs, and it is well tolerated by patients. ${ }^{27,28}$ Here, inhibition of GSH synthesis by BSO was shown to overcome cisplatin resistance in glioma cells, mainly because of an increase in DNA damage induction by cisplatin.

The $T M Z$ regimen has been shown to be effective and has rapidly replaced alkylating agents such as $\mathrm{BCNU}$; therefore, it was adopted as the first-line chemotherapeutic agent for patients with newly diagnosed glioblastoma. In fact, glioma patients responded well to TMZ and, when administered in combination with radiotherapy, a significant improvement in the 2-year survival rate was observed (27\% versus $10 \%) .{ }^{29}$ Similar to cisplatin, TMZ also targets DNA, producing several types of base lesions through methylation, and the alkylated product $\mathrm{O}^{6}$-methylguanine $\left(\mathrm{O}^{6} \mathrm{MeG}\right)$ is particularly harmful to the cells. This type of lesion is directly repaired by a suicide enzyme known as MGMT, which removes the methyl group of the DNA base and transfers it to its cysteine residue. ${ }^{30}$ As a consequence, the MGMT enzyme has a critical role in determining TMZ resistance; therefore, methylation of the promoter of the MGMT gene, which reduces its expression, is considered to be an important biomarker for drug responsiveness. ${ }^{30}$

In fact, Stupp et al. ${ }^{31}$ showed a strong correlation between MGMT promoter methylation and the effectiveness of $\mathrm{TMZ}$ treatment in a randomized, phase III trial. Interestingly, it has been shown that cisplatin treatment can reduce MGMT activity and expression, which is an explanation for the synergism between $\mathrm{TMZ}$ and cisplatin. ${ }^{32}$ Many clinical trials report the combination of $\mathrm{TMZ}$ and cisplatin as a valuable alternative for treating refractory and recurrent glioma. ${ }^{33-35}$

In the present work, we demonstrated that cellular resistance to $T M Z$ was highly overcome by $B S O$, whereas NAC prevented TMZ cytotoxicity. This is in agreement with previous work indicating that $\mathrm{TMZ}$ resistance in glioma is associated with lower production of reactive oxygen species by the resistant cell line, ${ }^{15}$ although this does not exclude the possibility of a scavenging action of GSH also for TMZ. Recent work has also indicated that BSO can potentiate the toxicity of TMZ-induced bystander effect on glioblastoma cells ${ }^{36}$ and a GSH transferase inhibitor was shown to potentiate the cytotoxic effect of TMZ in melanoma cells. ${ }^{37}$ In this work, we further demonstrated that in GSH-depleted cells, TMZ induces a higher amount of DNA damage. Thus, as TMZ generates the DNA-methylating carbenium ions, these may be scavenged by $\mathrm{SH}$-group-containing molecules, such as GSH, and this may be an important mechanism of $\mathrm{TMZ}$ detoxificaton within the cells.

Moreover, GSH depletion by BSO could strongly potentiate cellular death triggered by $\mathrm{TMZ}$ and cisplatin. Thus, the use of cisplatin and TMZ in low doses may attenuate the side effects caused by those drugs, and once combined, BSO, cisplatin and TMZ revealed to have a synergistic effect on glioma cells, which could improve the clinical outcome in patients.

Collectively, the data presented here indicated that a high intracellular GSH concentration protects against the killing effects of cisplatin. For the first time, GSH depletion associated with a drug treatment regimen with cisplatin and $\mathrm{TMZ}$ was demonstrated to substantially enhance cytotoxicity against glioma cells. Therefore, the intracellular GSH concentration is proposed as a potential molecular marker for cisplatin resistance in glioma, and the use of GSH inhibitors, such as BSO, in association with cisplatin and TMZ seems a promising approach for the therapy of this tumor.

\section{Materials and Methods}

Cell lines and culture conditions. Certified glioma cell lines U87MG and U343MG (p53 wild-type), U251MG (273Arg-His homozygous p53 mutation) and U138MG (273Arg-His heterozygous p53 mutation) were a kind gift from Bernd Kaina (Mainz, Germany), and were routinely grown in DMEM (Invitrogen, Life Technologies, Carlsbad, CA, USA) supplemented with $10 \%$ fetal calf serum (Cultilab, Campinas, Brazil) and $1 \%$ antibiotic-antimycotic at $37^{\circ} \mathrm{C}$ in a humidified, $5 \% \mathrm{CO}_{2}$ atmosphere.

Flow cytometry for sub-G1 and $\mathrm{\gamma H}_{2} \mathrm{AX}$ analysis. The apoptotic response after genotoxic drug treatment was measured using the flow cytometric method of sub-G1 determination. Supernatant and attached cells were collected, washed once with PBS and fixed in $70 \%$ ethanol. Ethanol-fixed cells were stained with propidium iodide (PI) at room temperature for $1 \mathrm{~h}$ in PBS containing $20 \mu \mathrm{g} / \mathrm{ml}$ PI (Sigma-Aldrich, St. Louis, MO, USA), $200 \mu \mathrm{g} / \mathrm{ml}$ RNase A and 0.1\% Triton X-100. The percentage of sub-G1 cells was calculated using the CytoSoft software (Millipore, Darmstadt, Germany). For $\gamma \mathrm{H}_{2} \mathrm{AX}$ immunostaining, cells were fixed with $1 \%$ formaldehyde and then with $70 \%$ ethanol. Afterwards, the cells were blocked, permeabilized and incubated with primary mouse monoclonal antibody to $\gamma \mathrm{H} 2 \mathrm{AX}$ (Ser-139) at 1: 1000 (Upstate Biotechnology, Upstate, NY, USA) and diluted 1:500 for $1 \mathrm{~h}$ at room temperature, followed by incubation with anti-mouse FITC secondary antibody (Sigma-Aldrich) that was diluted 1:200 for $1 \mathrm{~h}$ at room temperature.

Immunofluorescence microscopy. Cells were plated onto coverslips and placed in a $60 \mathrm{~mm}$ plate. The next day, the cells were treated with $10 \mu \mathrm{M}$ cisplatin for $3 \mathrm{~h}$, fixed with $4 \%$ formaldehyde, permeabilized with $0.5 \%$ Triton X-100 and blocked with $0.5 \%$ BSA and $0.3 \%$ Triton-X-100 in PBS. Thereafter, the cells were incubated with primary mouse monoclonal antibody to H2AX (Ser-139) diluted 1:1000 (Upstate Biotechnology) or primary antibody anti-cisplatin (CP9/19) (Abcam, Cambridge, MA, USA) that was diluted $1: 5000$ for $16 \mathrm{~h}$ at $4{ }^{\circ} \mathrm{C}$. Secondary antibodies labeled with Alexa 546 (Molecular Probes, Life Technologies Carlsbad, CA, USA) or FITC (Sigma-Aldrich) were added at 1:2000 and incubated at room temperature for $2 \mathrm{~h}$. Slides were mounted with a mounting-solution reagent containing DAPI (Vector Lab, Burlingame, CA, USA) and then analyzed using a Zeiss Axiovert 200 fluorescence microscope with AXIOVISION 4.2 software (Carl Zeiss, Jena, Germany).

Western blot. For cellular protein detection, the following antibodies were used: mouse anti-p53 (Abcam), mouse p21 (Santa Cruz, Dallas, TX, USA), rabbit antiSLC31A1/CTR1 (Abcam), mouse anti-tubulin (Abcam) and mouse anti-GAPDH (Santa Cruz). 
Immunoblot assay. Following extraction of genomic DNA, the samples were denatured at $100^{\circ} \mathrm{C}$ for $10 \mathrm{~min}$, and $500 \mathrm{ng}$ per well was spotted onto nitrocellulose membranes (Bio-Rad, Hercules, CA, USA) using a slot-blot apparatus (Omniphor, San Jose, CA, USA). The membrane was baked for $2 \mathrm{~h}$ at $80^{\circ} \mathrm{C}$ and then blocked in $5 \%$ milk that was diluted in a PBS buffer for $1 \mathrm{~h}$ at room temperature. Subsequently, the membranes were incubated with anti-cisplatin (CP9/19) primary antibody (Abcam) that was diluted 1:5000 in 5\% milk-PBS overnight at $4{ }^{\circ} \mathrm{C}$. The secondary antibody, anti-mouse IgG HRP conjugate (R\&D Systems, Minneapolis, MN, USA), was diluted 1:2000 in $5 \%$ milk-PBS, and the membranes were incubated for $2 \mathrm{~h}$ at room temperature. DNA lesions were detected by adding the chemiluminescence reagent Luminata western HRP substrate (Millipore), and using ImageQuant 300 (GE Healthcare, Little Chalfont, Buckinghamshire, UK).

HCR assay. The mammalian expression vectors $p S h u t t l e / L u c$ and $p S h u t t l e / R L$ were generated as described previously. ${ }^{38}$ PShuttle/Luc was treated with 10-500 $\mathrm{nM}$ cisplatin for $4 \mathrm{~h}$ at $40^{\circ} \mathrm{C}$ to induce DNA damage, and $1 \times 10^{4}$ cells were plated in 96-well dishes, in triplicate, for each point. A total of $200 \mathrm{ng}$ of plasmids (180 ng pShuttle/Luc and $20 \mathrm{ng}$ pShuttle/RL) was used for transfection using Lipofectamine 2000 Transfection Reagent (Invitrogen, Life Technologies). Two days after DNA transfection, luciferase activities were measured using the Dual-Glo Luciferase Assay System (Promega, Madison, WI, USA) and a Glomax-Multi+ Luminometer (Promega).

GSH quantification. The cells were scrapped and pelleted by centrifugation at 1500 r.p.m. for $10 \mathrm{~min}$. Then, the cells were incubated on ice in a 1: 10 PBS mobile phase solution containing metaphosphoric acid $5 \%$ for $30 \mathrm{~min}$. GSH content was investigated using an HPLC coupled to the electrochemical detection (ESA Thermo Scientific, Sunnyvale, CA, USA) system. The equipment was composed of 584 pumps, CoulArray detector $5600 \mathrm{~A}$ and UV-Vis detector SPD-10AV VP (Shimadzu, Kyoto, Japan) and was used with a Kinetix column C18 $100 \times 2.1 \mathrm{~mm}^{2}, 2.6 \mu \mathrm{m}$ (Phenomenex, Torrance, CA, USA) maintained at a temperature of $28^{\circ} \mathrm{C}$. The mobile phase consisted of sodium phosphate $25 \mathrm{mM}$, octassulfonic acid $50 \mu \mathrm{M}, \mathrm{pH}$ 2.6, and acetonitrile 1\%. Each run was performed on an isocratic flow (0.4 $\mathrm{ml} \times \mathrm{min}^{-1}$ ) for $10 \mathrm{~min}$. The data were collected at $215 \mathrm{~nm}$ in an UV detector, and at 400,600 and $950 \mathrm{mV}$ in an electrochemical detector.

Also, cells were seeded in opaque, 96-well plates and allowed to grow for $24 \mathrm{~h}$ under cell culture conditions. After $18 \mathrm{~h}$ of incubation with $100 \mu \mathrm{M} \mathrm{BSO}$ or $25 \mathrm{mM}$ NAC, the intracellular GSH levels were quantified using the GSH-Glo GSH Assay (Promega), as recommended by the manufacturer's instructions. Briefly, cells were washed with PBS and incubated for $30 \mathrm{~min}$ at room temperature in a solution containing luciferin NT substrate and GST. Then, $100 \mu$ l of luciferase enzyme was added and incubated for $15 \mathrm{~min}$ at room temperature. Luminescence was measured using a Glomax-Multi+ Luminometer (Promega). In both methods serial dilutions of a GSH standard solution were used to generate a standard curve, and the GSH concentration was normalized to the protein concentration of each well.

Quantitative PCR (QPCR). The quantitative PCR (QPCR) reaction was performed as described previously. ${ }^{12}$ Briefly, after genomic DNA extraction and quantification by PicoGreen fluorescence (Invitrogen, Carlsbad, CA, USA), the DNA was used as template for the PCR reaction. For these, TaKaRa LA PCR (Takara Bio Inc., Mountain View, CA, USA) and the sequencing primers $5^{\prime}$-TGGAAA CCCTGTGGGCGGATAATA-3' and 5'-CTCCAGGCCTAAGGAGCAGCAGAA-3' were used. The PCR products were quantified by PicoGreen fluorescence, and the cisplatintreated $(50 \mu \mathrm{M})$ or BSO-treated samples $(100 \mathrm{mM})$ were divided by control sample. The resulting ratio was the relative amplification of damaged to control samples, and the DNA lesion frequencies were estimated based on the Poisson distribution.

Establishment of glioma expressing luciferase. The luciferase gene reporter (Luc) was cloned into the Xhol site of the lentiviral vector PLV-CMV-SV40Puro (kindly provided by Prof. Dr. Silvya Engler, University of São Paulo, São Paulo, Brazil), generating $\mathrm{pLV} / \mathrm{L} u c$. This plasmid was co-transfected with three auxiliary plasmids into HEK $293 \mathrm{~T}$ cells using the polyethyleneimine method. The recombinant lentivirus was then used to transduce U87MG and U138MG cells, and selection was performed with incubation for 2 weeks with puromycin $(1.5 \mu \mathrm{g} /$ $\mathrm{ml})$, resulting in the stable-expressing luciferase glioma cell lines U87-Luc and U138-Luc.
In vivo procedures. Xenograft tumors were established in 10-12-week-old, female, athymic nude mice. U87-Luc cells $\left(3 \times 10^{6}\right)$ were inoculated subcutaneously in the animal's flank. Tumors were allowed to grow, and $\sim 3$ weeks after inoculation, treatment began. Tumor volume measurements were calculated according to the following formula: volume $=\left(\right.$ width $^{2} \times$ length $) / 2$. The animals were randomized into eight treatment groups: (1) PBS; (2) BSO $(450 \mathrm{mg} / \mathrm{kg})$; (3) cisplatin (1 mg/kg); (4) TMZ (10 mg/kg); (5) TMZ+cisplatin; (6) BSO+cisplatin; (7) BSO+TMZ; and (8) BSO+cisplatin+TMZ. BSO was inoculated intraperitoneally $5 \mathrm{~h}$ before cisplatin or TMZ treatment for three consecutive days and then once a week for 2 weeks. All animal procedures were approved by the Ethics Animal Care and Use Committee of the Institute of Biomedical Sciences, University of Sao Paulo.

Bioluminescence imaging. For in vitro luciferase assays, cells were plated in 12-well plates, allowed to attach overnight and then treated with BSO $(100 \mu \mathrm{M})$, cisplatin $(1 \mu \mathrm{M})$ and/or TMZ $(10 \mu \mathrm{M})$. After $120 \mathrm{~h}$, the medium was replaced by fresh medium containing D-luciferin $(150 \mu \mathrm{g} / \mathrm{ml})$. For in vivo assays, D-luciferin $(150 \mathrm{mg} / \mathrm{kg})$ was inoculated intraperitoneally into the nude mice to measure the tumor size, and Z-DEVD-aminoluciferin $(50 \mathrm{mg} / \mathrm{kg})$ was used to detect apoptosis. Bioluminescence images were obtained using the IVIS Spectrum System (Perkin-Elmer Life Sciences, Waltham, MA, USA) at the CEFAP-USP facility.

Statistical analysis. Results represent the mean of three independent experiments, each performed in triplicate, with error bars showing the S.E.M. Statistical significance was assessed using one-way ANOVA followed by Bofferoni posttesting (GraphPad Prism 6; GraphPad Software Inc., San Diego, CA, USA) $\left({ }^{\star} P<0.01,{ }^{* \star} P<0.005\right.$ and $\left.{ }^{* \star *} P<0.001\right)$.

\section{Conflict of Interest}

The authors declare no conflict of interest.

Acknowledgements. We are grateful for the excellent technical assistance provided by the Core Facility for Scientific Research-USP (CEFAP-USP) in conducting animal imaging with the IVIS Spectrum system. We also thank Dr. Marisa Medeiros for helping to conduct the GSH quantification. This work was supported by Fundação de Amparo à Pesquisa do Estado de São Paulo (FAPESP) (Sao Paulo, Brazil), CAPES and Conselho Nacional de Desenvolvimento Científico e Tecnológico (CNPq) (Brasilia, Brazil).

1. Wen PY, Kesari S. Malignant gliomas in adults. N Engl J Med 2008; 359: 492-507.

2. Beier D, Schulz J, Beier C. Chemoresistance of glioblastoma cancer stem cells -much more complex than expected. Mol Cancer 2011; 10: 128.

3. Johannessen T-CA, Bjerkvig R. Molecular mechanisms of temozolomide resistance in glioblastoma multiforme. Expert Rev Anticancer Ther 2012; 12: 635-642.

4. Roos WP, Batista LFZ, Naumann SC, Wick W, Weller M, Menck CFM et al. Apoptosis in malignant glioma cells triggered by the temozolomide-induced DNA lesion 0 6 -methylguanine. Oncogene 2007; 26: 186-197.

5. Batista LFZ, Roos WP, Christmann M, Menck CFM, Kaina B. Differential sensitivity of malignant glioma cells to methylating and chloroethylating anticancer drugs: p53 determines the switch by regulating xpc, ddb2, and DNA double-strand breaks. Cancer Res 2007; 67: 11886-11895.

6. Kelland L. The resurgence of platinum-based cancer chemotherapy. Nat Rev Cancer 2007; 7: $573-584$.

7. Van den Bent MJ, Hegi ME, Stupp R. Recent developments in the use of chemotherapy in brain tumours. Eur J Cancer 2006; 42: 582-588.

8. Galluzzi L, Senovilla L, Vitale I, Michels J, Martins I, Kepp O et al. Molecular mechanisms of cisplatin resistance. Oncogene 2012; 31: 1869-1883.

9. Byun S-S, Kim SW, Choi H, Lee C, Lee E. Augmentation of cisplatin sensitivity in cisplatinresistant human bladder cancer cells by modulating glutathione concentrations and glutathione-related enzyme activities. BJU Int 2005; 95: 1086-1090.

10. Hoebers FJP, Pluim D, Verheij M, Balm AJM, Bartelink H, Schellens JHM et al. Prediction of treatment outcome by cisplatin-DNA adduct formation in patients with stage III/IV head and neck squamous cell carcinoma, treated by concurrent cisplatin-radiation (RADPLAT). Int J Cancer 2006; 119: 750-756.

11. Ali-Osman F, Brunner JM, Kutluk TM, Hess K. Prognostic significance of glutathione S-transferase pi expression and subcellular localization in human gliomas. Clin Cancer Res 1997; 3: 2253-2261. 
12. Olive PL, Banáth JP. Kinetics of H2AX phosphorylation after exposure to cisplatin. Cytom Part B 2009; 76B: 79-90.

13. Cleaver JE. $\gamma \mathrm{H} 2 \mathrm{Ax}$ : biomarker of damage or functional participant in dna repair 'All that Glitters Is not Gold!'. Photochem Photobiol 2011; 87: 1230-1239.

14. Santos J, Meyer J, Mandavilli B, Houten B. Quantitative PCR-based measurement of nuclear and mitochondrial DNA damage and repair in mammalian cells. DNA Repair 2006: 183-199.

15. Oliva CR, Moellering DR, Gillespie GY, Griguer CE. Acquisition of chemoresistance in gliomas is associated with increased mitochondrial coupling and decreased ROS production. PLoS One 2011; 6: e24665.

16. Kim CK, Nguyen TLX, Joo KM, Nam D-H, Park J, Lee K-H et al. Negative regulation of p53 by the long isoform of ErbB3 binding protein Ebp1 in brain tumors. Cancer Res 2010; 70: 9730-9741.

17. Datta K, Shah P, Srivastava T, Mathur SG, Chattopadhyay P, Sinha S. Sensitizing glioma cells to cisplatin by abrogating the p53 response with antisense oligonucleotides. Cancer Gene Ther 2004; 11: 525-531.

18. Tallen U, Truss M, Kunitz F, Wellmann S, Unryn B, Sinn B et al. Down-regulation of the inhibitor of growth 1 (ING1) tumor suppressor sensitizes p53-deficient glioblastoma cells to cisplatin-induced cell death. J Neurooncol 2008; 86: 23-30.

19. Park CM, Park MJ, Kwak HJ, Moon SI, Yoo DH, Lee HC et al. Induction of p53-mediated apoptosis and recovery of chemosensitivity through p53 transduction in human glioblastoma cells by cisplatin. Int J Oncol 2006; 28: 119-125.

20. Martin LP, Hamilton TC, Schilder RJ. Platinum resistance: the role of DNA repair pathways. Clin Cancer Res 2008; 14: 1291-1295.

21. Parker RJ, Eastman A, Bostick-Bruton F, Reed E. Acquired cisplatin resistance in human ovarian cancer cells is associated with enhanced repair of cisplatin-DNA lesions and reduced drug accumulation. J Clin Invest 1991; 87: 772-777.

22. Ferry KV, Hamilton TC, Johnson SW. Increased nucleotide excision repair in cisplatinresistant ovarian cancer cells: role of ercc1-xpf. Biochem Pharmacol 2000; 60: 1305-1313.

23. Selvakumaran M, Pisarcik DA, Bao R, Yeung AT, Hamilton TC. Enhanced cisplatin cytotoxicity by disturbing the nucleotide excision repair pathway in ovarian cancer cell lines. Cancer Res 2003; 63: 1311-1316.

24. Chen HHW, Kuo MT. Role of glutathione in the regulation of Cisplatin resistance in cancer chemotherapy. Met Based Drugs 2010; 2010: 430939.

25. Van de Vaart PJ, Belderbos J, de Jong D, Sneeuw KC, Majoor D, Bartelink $\mathrm{H}$ et al. DNA-adduct levels as a predictor of outcome for NSCLC patients receiving daily cisplatin and radiotherapy. Int J Cancer 2000; 89: 160-166.

26. Rudin CM, Yang Z, Schumaker LM, VanderWeele DJ, Newkirk K, Egorin MJ et al. Inhibition of glutathione synthesis reverses Bcl-2-mediated cisplatin resistance. Cancer Res 2003; 63 : 312-318.

27. O'Dwyer PJ, Hamilton TC, LaCreta FP, Gallo JM, Kilpatrick D, Halbherr T et al. Phase I trial of buthionine sulfoximine in combination with melphalan in patients with cancer. $\mathrm{J}$ Clin Oncol 1996; 14: 249-256.

28. Calvert P, Yao K-S, Hamilton TC, O'Dwyer PJ. Clinical studies of reversal of drug resistance based on glutathione. Chem Biol Interact 1998; 111: 213-224.
29. Stupp R, Mason WP, van den Bent MJ, Weller M, Fisher B, Taphoorn MJB et al. Radiotherapy plus concomitant and adjuvant temozolomide for glioblastoma. $N$ Engl J Med 2005; 352: 987-996.

30. Pegg AE. Repair of O6-alkylguanine by alkyltransferases. Mutat Res 2000; 462: 83-100.

31. Stupp R, Hegi ME, Mason WP, van den Bent MJ, Taphoorn MJB, Janzer RC et al. Effects of radiotherapy with concomitant and adjuvant temozolomide versus radiotherapy alone on survival in glioblastoma in a randomised phase III study: 5-year analysis of the EORTC-NCIC trial. Lancet Oncol 2009; 10: 459-466.

32. D'Atri S, Graziani G, Lacal PM, Nisticò V, Gilberti S, Faraoni I et al. Attenuation of 06-methylguanine-DNA methyltransferase activity and mRNA levels by cisplatin and temozolomide in jurkat cells. J Pharmacol Exp Ther 2000; 294 664-671.

33. Brandes AA, Basso U, Reni M, Vastola F, Tosoni A, Cavallo G et al. First-line chemotherapy with cisplatin plus fractionated temozolomide in recurrent glioblastoma multiforme: A Phase II Study of the Gruppo Italiano Cooperativo di Neuro-Oncologia. J Clin Oncol 2004; 22: 1598-1604.

34. Zustovich F, Lombardi G, Della Puppa A, Rotilio A, Scienza R, Pastorelli D. A phase II study of cisplatin and temozolomide in heavily pre-treated patients with temozolomide-refractory high-grade malignant glioma. Anticancer Res 2009; 29 4275-4279.

35. Silvani A, Eoli M, Salmaggi A, Lamperti E, Maccagnano E, Broggi G et al. Phase II trial of cisplatin plus temozol omide, in recurrent and progressive malignant glioma patients. J Neurooncol 2004; 66: 203-208.

36. Kohsaka S, Takahashi K, Wang L, Tanino M, Kimura T, Nishihara H et al. Inhibition of GSH synthesis potentiates temozolomide-induced bystander effect in glioblastoma. Cancer Lett 2013; 331: 68-75

37. Tentori L, Dorio AS, Mazzon E, Muzi A, Sau A, Cuzzocrea S et al. The glutathione transferase inhibitor 6-(7-nitro-2,1,3-benzoxadiazol-4-ylthio)hexano (NBDHEX) increases temozolomide efficacy against malignant melanoma. Eur J Cancer 2011; 47: 1219-1230.

38. Soltys DT, Rocha CRR, Lerner LK, de Souza TA, Munford VCabral F et al. Novel XPG (ERCC5) mutations affect DNA repair and cell survival after ultraviolet but not oxidative stress. Hum Mutat 2013; 34: 481-489.

(i)

Cell Death and Disease is an open-access journal published by Nature Publishing Group. This work is licensed under a Creative Commons Attribution 4.0 International Licence. The images or other third party material in this article are included in the article's Creative Commons licence, unless indicated otherwise in the credit line; if the material is not included under the Creative Commons licence, users will need to obtain permission from the licence holder to reproduce the material. To view a copy of this licence, visit http://creativecommons.org/licenses/by/4.0 